\title{
Bicalutamide-induced hypoxia potentiates RUNX2-mediated Bcl-2 expression resulting in apoptosis resistance
}

\author{
G Browne',2, H Nesbitt', L Ming', GS Stein², JB Lian², SR McKeown' and J Worthington*,1 \\ 'Biomedical Science Research Institute, University of Ulster, Cromore Road, Coleraine, Londonderry BT52 ISA, Northern Ireland; ${ }^{2}$ Department of Cell \\ Biology and Cancer Center, University of Massachusetts Medical School, Worcester, MA, USA
}

BACKGROUND: We have previously shown that hypoxia selects for more invasive, apoptosis-resistant LNCaP prostate cancer cells, with upregulation of the osteogenic transcription factor RUNX2 and the anti-apoptotic factor Bcl-2 detected in the hypoxia-selected cells. Following this observation, we questioned through what biological mechanism this occurs.

METHODS: We examined the effect of hypoxia on RUNX2 expression and the role of RUNX2 in the regulation of Bcl-2 and apoptosis resistance in prostate cancer.

RESULTS: Hypoxia increased RUNX2 expression in vitro, and bicalutamide-treated LNCaP tumours in mice (previously shown to have increased tumour hypoxia) exhibited increased RUNX2 expression. In addition, RUNX2-overexpressing LNCaP cells showed increased cell viability, following bicalutamide and docetaxel treatment, which was inhibited by RUNX2 siRNA; a range of assays demonstrated that this was due to resistance to apoptosis. RUNX2 expression was associated with increased Bcl-2 levels, and regulation of $\mathrm{Bcl}-2$ by RUNX2 was confirmed through chromatin immunoprecipitation (ChIP) binding and reporter assays. Moreover, a Q-PCR array identified other apoptosis-associated genes upregulated in the RUNX2-overexpressing LNCaP cells.

CONCLUSION: This study establishes a contributing mechanism for progression of prostate cancer cells to a more apoptosis-resistant and thus malignant phenotype, whereby increased expression of RUNX2 modulates the expression of apoptosis-associated factors, specifically Bcl-2.

British Journal of Cancer (2012) I 07, 17|4-172I. doi:I0.1038/bjc.2012.455 www.bjcancer.com

Published online 16 October 2012

(C) 2012 Cancer Research UK

Keywords: hypoxia; RUNX2; Bcl-2; bicalutamide; prostate cancer; LNCaP

Prostate cancer remains a prevalent disease among males, with nearly 900000 cases recorded worldwide in 2008 (Ferlay et al, 2010). Androgen-deprivation therapy (ADT) is commonly used as a first-line treatment for prostate cancer; however, recent studies have indicated that ADT can result in profound hypoxia (oxygen deprivation) in prostate tumours (Alonzi et al, 2011; Ming et al, 2012). This is clinically important because hypoxia is known to be a significant physiological stress in the tumour microenvironment, encouraging progression and therapeutic resistance of many cancers, including prostate cancer (Chaudary and Hill, 2007). Much evidence has indicated that hypoxia (both chronic and acute/cyclic) affects various aspects of tumorigenesis frequently through induction of the hypoxia-inducible factor (HIF) transcription factors (Majmundar et al, 2010). These regulatory mechanisms have recently been reviewed by others (Cassavaugh and Lounsbury, 2011). In the present study, we identified mechanistic links between hypoxia, RUNX2, a key transcription factor related to aggressive prostate cancer, and apoptosis resistance.

RUNX2 is associated with tumour growth, invasion and metastasis (Pratap et al, 2006; Zhang et al, 2011). In prostate cancer, increased expression of RUNX2 was shown to activate numerous genes associated with tumour growth and invasion (e.g., VEGF and MMPs), as well as to correlate positively with

*Correspondence: Dr J Worthington; E-mail: J.Worthington@ulster.ac.uk Received 3 May 2012; revised 10 September 2012; accepted 12 September 2012; published online 16 October 2012
Gleason scores in human prostate cancer tissue samples (Chua et al, 2009; Akech et al, 2010). Recent studies have shown that RUNX2 physically and functionally interacts with both HIF1A and HIF-2A (Tamiya et al, 2008; Kwon et al, 2011), indicating that hypoxia may be an important regulator of RUNX2. Moreover, Butterworth et al (2008) found that hypoxia selects for more invasive, apoptosis-resistant prostate cancer cells, which have increased RUNX2 and Bcl-2 expression.

Apoptosis evasion is one of the hallmarks of cancer, and disruption of normal apoptotic regulatory mechanisms in cancer cells can be a major molecular force driving the progression of the disease (Hanahan and Weinberg, 2011). Upregulated expression of Bcl-2 has been found to be a feature of many cancers, including prostate cancer, and is associated with more aggressive disease and resistance to chemotherapy (Bonkhoff and Berges, 2010). A role for RUNX2 in apoptosis was first identified by Bellido et al (2003), who showed that the anti-apoptotic effect of parathyroid hormone was mediated by RUNX2. It was also found that RUNX2expressing lymphomas have low apoptotic rates even in the presence of Myc overexpression (Blyth et al, 2006).

In this study, we found that anti-androgen therapy increases RUNX2 expression in prostate cancer cells. Both in vitro and in vivo analyses demonstrate that hypoxia promotes overexpression of RUNX2 at the transcriptional level and that prostate cancer cells with increased RUNX2 expression exhibit a survival advantage by increasing $\mathrm{Bcl}-2$ expression through direct regulation of its promoter activity. We established that this increased RUNX2 
expression promotes progression to a more apoptosis-resistant and thus a malignant phenotype. These results have delineated a contributing mechanism of prostate cancer progression and strengthen the argument for RUNX2 as a viable therapeutic target.

\section{MATERIALS AND METHODS}

\section{Cell culture}

Parental LNCaP prostate adenocarcinoma cells (ATCC, Rockville, MD, USA) and generated sublines were maintained in RPMI-1640 medium supplemented with $10 \%$ fetal bovine serum (FBS), $10 \mathrm{~mm}$ HEPES and $5 \mathrm{~mm}$ glucose. PC3 prostate carcinoma cells (ATCC) were maintained in RPMI-1640 medium supplemented with $10 \%$ FBS. Cells were cultured at $37^{\circ} \mathrm{C}$ under $5 \% \mathrm{CO}_{2}$ in air. For experiments that required hypoxic conditions, the In $\mathrm{Vivo}_{2}$ Hypoxic workstation (Ruskinn Technologies, Bridgend, UK) was used.

\section{LNCaP xenograft model}

All the experiments were conducted in accordance with the Animal (Scientific Procedures) Act 1986 and the UKCCCR guidelines (2010) for the welfare of animals in experimental neoplasia (Workman et al, 2010). LNCaP cells $\left(5 \times 10^{6}\right.$ in matrigel) were implanted on the rear dorsum of 8-10-week-old male severe immunocompromised (SCID) mice. When tumours reached $150-200 \mathrm{~mm}^{3}$, mice were treated by gavage with vehicle only ( $0.1 \%$ DMSO in corn oil; both Sigma, UK) or the anti-androgen bicalutamide $\left(2 \mathrm{mg} \mathrm{kg}^{-1}\right.$ per day; AstraZeneca, Macclesfield, UK) for 28 days.

\section{Immunohistochemistry}

Animals were treated with bicalutamide or vehicle and killed after $0,7,14,21$ and 28 days of treatment. Tumours were removed and fixed in $4 \%$ formalin (Sigma) before paraffin embedding and sectioning at $5 \mu \mathrm{M}$. Sections were stained with RUNX2 (M70) primary antibody (1:50; Santa Cruz Biotechnology, Heidelberg, Germany) as described in Supplementary Methods. Five regions of interest from three slides per tumour and three individual tumours were quantified. Regions of interest were the centre of the tumour section and each of the surrounding areas immediately north, south, east and west of this. Each slide was scored in a blind manner by two independent observers and the results averaged.

\section{Gene expression analysis (RT-Q-PCR)}

RNA was extracted from cells using the High Pure RNA Isolation kit (Roche, Welwyn Garden City, UK) and reverse transcribed using RevertAid reagents (Fermentas, Cambridge, UK). Primer sequences can be found in Supplementary Methods. Samples from three independent experiments were analysed in triplicate. For the RT-Q-PCR array, the RealTime Ready Human Apoptosis Panel (Roche, UK) was used according to the manufacturer's instructions.

\section{Western blotting}

A detailed description of western blotting of whole-cell lysates extracted in RIPA buffer can be found in Supplementary Methods.

\section{RNA stability assay}

LNCaP cells were exposed to hypoxia $\left(0.1 \% \mathrm{O}_{2}\right)$ for $2 \mathrm{~h}$ before adding actinomycin D (Sigma) to the culture medium to a final concentration of $5 \mu \mathrm{g} \mathrm{ml}^{-1}$. Cells were returned to hypoxia for the duration of the experiment. RNA was extracted from cells at $0,2,4$, 6 and $8 \mathrm{~h}$ following treatment, cDNA prepared and expression of RUNX2 mRNA assessed using Q-PCR.

\section{Generation of stable RUNX2-overexpressing cells}

LNCaP sublines were generated by either lentiviral gene delivery as reported previously (Leong et al, 2010) or by stably transfecting with either empty vector to create $\mathrm{LNCaP}-\mathrm{V}$ or vector containing RUNX2 to create LNCaP-R using Lipofectamine 2000 (Invitrogen, Paisley, UK). Confirmation of RUNX2 expression was carried out using RT-Q-PCR and western blotting.

\section{XTT assay}

Cells were plated at $1 \times 10^{4}$ into a 96-well plate and left overnight to adhere. Docetaxel (in $0.001 \%$ DMSO) was added $(1,2,10,25,50$ and $100 \mathrm{~nm}$ final concentration) and cells were incubated at $37^{\circ} \mathrm{C}$ for $48 \mathrm{~h}$. XTT assay was carried out (Roche, UK) with absorbance measured at $490 \mathrm{~nm}$ using a Versamax plate reader (Molecular Devices Inc., Sunnyvale, CA, USA).

\section{DNA laddering assay}

Cells $\left(2 \times 10^{6}\right.$ per dish) were plated in $100 \mathrm{~mm}$ culture dishes and left to adhere overnight before treating with $100 \mathrm{~nm}$ docetaxel for 0 , 24,36 or $48 \mathrm{~h}$. Then, $2 \times 10^{6}$ cells from each treatment group were resuspended in PBS and DNA was extracted using the Apoptotic DNA-ladder kit (Roche, Indianapolis, IN, USA). The band intensities of the resultant ladders were quantified using GelQuant.NET software provided by biochemlabsolutions.com.

\section{Caspase 3/7 luminescent assay}

Cells $\left(1 \times 10^{4}\right.$ per well) were seeded in a 96-well plate and left to adhere for overnight before treating with $100 \mathrm{~nm}$ docetaxel for $48 \mathrm{~h}$. Following treatment, $\mathrm{LNCaP}-\mathrm{V}$ and $\mathrm{R}$ cells were subjected to caspase $3 / 7$ activity measurement with Caspase-Glo assay kit (Promega, Madison, WI, USA). Luminescence was measured using a GLOMAX luminometer (Promega).

\section{TUNEL assay}

The TUNEL assay was performed using the Click-It TUNEL kit (Invitrogen). Briefly, $5 \times 10^{5}$ cells were seeded on individual microscopy slides and allowed to adhere overnight before treatment with $100 \mathrm{~nm}$ docetaxel for the indicated times. Post processing, cells were visualised using a Nikon Eclipse E400 (Nikon UK, Surrey, UK) inverted fluorescent microscope. Analysis of TUNEL-positive cells was carried out on five fields of view on each of the three slides in three independent experiments.

\section{Caspase 3 cleavage assay}

Cells $\left(2 \times 10^{6}\right.$ per dish) were plated in $100-\mathrm{mm}$ culture dishes and left to adhere overnight before treating with $100 \mathrm{~nm}$ docetaxel for times indicated. Subsequently, whole-cell lysates were subjected to western blotting (above) and probed for cleaved caspase 3 protein.

\section{SiRNA}

Cells were transfected with RUNX2 or Bcl-2 siRNA (100 nM; both Santa Cruz Biotechnology) using siRNA Transfection Reagent (Santa Cruz Biotechnology). Confirmation of knockdown was carried out using RT-Q-PCR and western blotting. Control siRNA-A (100 nм; Santa Cruz Biotechnology) was used as a negative control.

\section{Luciferase assay}

A luciferase assay to assess Bcl-2 promoter activity was carried out using a Dual-Glo Luciferase Assay System (Promega, Southampton, UK). Detailed instructions can be found in Supplementary Methods. Mutation of the two putative RUNX2-binding sites within the Bcl-2 promoter was achieved using the Quikchange Site-Directed 
mutagenesis kit (Stragene, La Jolla, CA, USA) according to the manufacturer's instructions.

\section{ChIP assay}

The ChIP assay was performed on cells using the ChIP-IT Express kit (Actif Motif, La Hulpe, Belgium). In addition to analysis in LNCaP-V, LNCaP-R and PC3 cells (positive control), parental LNCaP cells were exposed to either oxic or hypoxic $\left(0.1 \% \mathrm{O}_{2}\right.$ for $\left.2 \mathrm{~h}\right)$ conditions before cross-linking to confirm RUNX2 occupancy on the Bcl-2 promoter under hypoxic conditions. A more detailed description of the ChIP assay can be found in Supplementary Methods.

\section{RESULTS}

\section{The anti-androgen bicalutamide modulates RUNX2} expression in LNCaP tumours in vivo

Because bicalutamide is commonly used to treat locally advanced prostate cancer, we examined the effect of a clinically relevant dose of bicalutamide ( $2 \mathrm{mg} \mathrm{kg}^{-1}$ per day) on RUNX2 expression in $\mathrm{LNCaP}$ tumours in vivo. Immunohistological examination of tumours revealed that RUNX2 expression increased with time in vehicle-treated animals (Figure 1A); this increase was much more pronounced in bicalutamide-treated animals. Bicalutamide treatment resulted in a significant increase (2.6 fold) in RUNX2expressing cells after 14 days (Figure $1 \mathrm{~B}, P \leqslant 0.01$ ). After 28 days, the number of tumour cells expressing RUNX2 protein was 3.5-fold higher than in vehicle-treated tumours. These results were confirmed by RT-Q-PCR analysis of RNA extracted from tumours (Figure $1 C, P \leqslant 0.05)$. To identify the mechanism of increased RUNX2 expression, we treated LNCaP cells in vitro with an equivalent dose of bicalutamide and measured the expression of RUNX2 mRNA; in vitro treatment of LNCaP cells with bicalutamide failed to induce RUNX2 expression (Figure 1D). This result, coupled with previous results from our laboratory showing increased tumour hypoxia in bicalutamide-treated LNCaP tumours (Ming et al, 2012), prompted us to investigate the effect of hypoxia on Runx2 expression in LNCaP cells. We found that both acute $\left(0.1 \% \mathrm{O}_{2}\right.$ for $\left.2 \mathrm{~h}\right)$ and chronic $\left(0.1 \% \mathrm{O}_{2}\right.$ for $\left.24 \mathrm{~h}\right)$ hypoxia

A Treatment time (days)

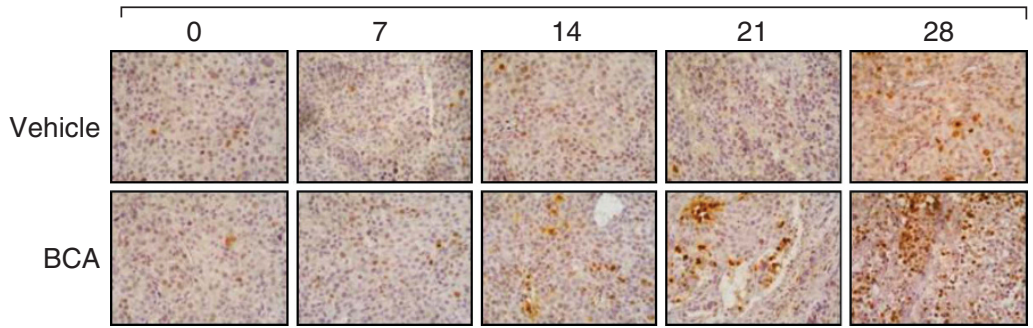

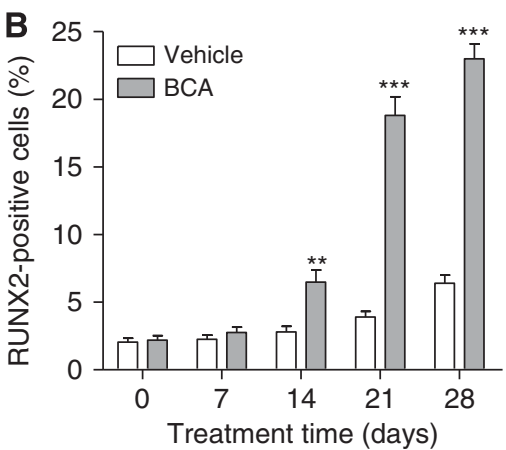

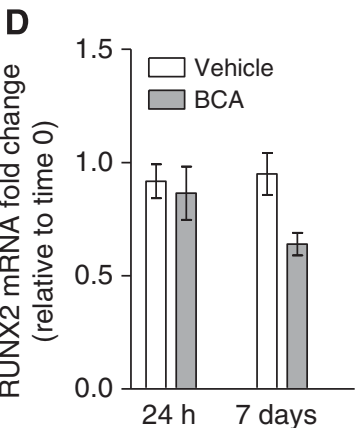

E

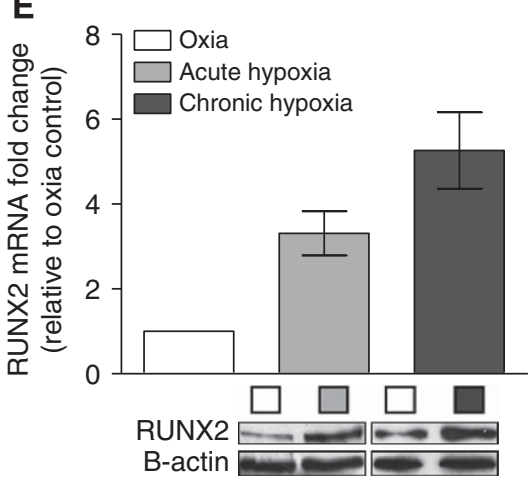

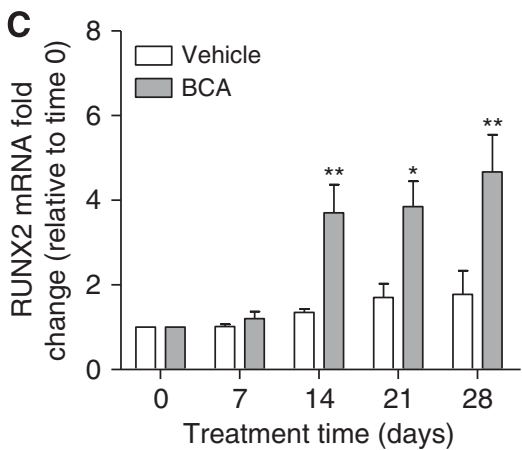

$\mathbf{F}$

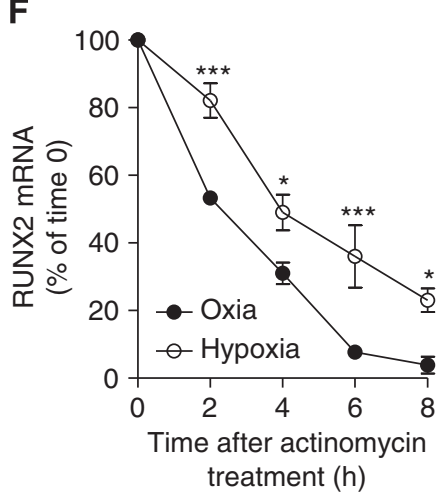

Figure I Bicalutamide (BCA) treatment increases RUNX2 expression in LNCaP tumours in vivo; hypoxia increases RUNX2 expression in LNCaP cells in vitro. (A) Representative images of RUNX2 staining in LNCaP tumours. Magnification $\times 400$. (B) Percentage of LNCaP cells staining positively for RUNX2. Results shown are mean \pm s.e. of five regions of interest for three slides for each of the three independent tumours. (C) RUNX2 mRNA fold change in tumours measured using RT-Q-PCR. Results shown are mean \pm s.e. of three independent tumours. (D) RUNX2 mRNA fold change in LNCaP cells treated with BCA in vitro (4.6 $\mu \mathrm{M})$. Results shown are mean \pm s.e. of four independent experiments. (E) RUNX2 mRNA expression in oxia and acute $\left(0.1 \% \mathrm{O}_{2}\right.$ for $\left.2 \mathrm{~h}\right)$ and chronic $\left(0.1 \% \mathrm{O}_{2}\right.$ for $\left.24 \mathrm{~h}\right)$ hypoxia. Results shown are mean \pm s.e. of three independent experiments. $(\mathbf{F})$ RUNX2 mRNA expression under oxic or hypoxic $\left(0.1 \% \mathrm{O}_{2}\right)$ conditions after exposure to actinomycin $D\left(5 \mu \mathrm{g} \mathrm{ml}^{-1}\right)$. Results shown are mean \pm s.e. of three independent experiments. ${ }^{*} P \leqslant 0.05,{ }^{* * *} P \leqslant 0.0$ I and ${ }^{* * *} P \leqslant 0.00$ I (two-way ANOVA). 
increased RUNX2 mRNA expression (Figure 1E). Exposure to hypoxia increased the half-life of RUNX2 mRNA from $2 \mathrm{~h}$ in oxia to $4.7 \mathrm{~h}$ (Figure $1 \mathrm{~F}$ ), indicating that the hypoxia-induced increase may be mediated by an increase in the half-life of RUNX2 mRNA.

Collectively, the in vitro and in vivo data presented suggest that the microenvironmental stress of hypoxia causes increased RUNX2 expression.

\section{RUNX2 influences cell survival}

Subsequently, we investigated whether RUNX2 may have a role in prostate cancer cell survival. Following failure of ADT, prostate cancer is treated with the chemotherapy agent docetaxel; thus, docetaxel was chosen as a clinically relevant apoptosis-inducing drug to use in subsequent experiments. We created LNCaP cell lines that expressed three times more RUNX2 protein than a vector-only control (Figure 2A; LNCaP-R and LNCaP-V, respectively). LNCaP-R cells were more resistant to docetaxel treatment than LNCaP-V cells in an XTT assay (Figure 2A). To confirm that increased cell viability following exposure to docetaxel was due to increased RUNX2, LNCaP-R cells were transfected with scrambled or RUNX2 siRNA, and cell viability was measured following docetaxel treatment (Figure 2B). Following 78\% knockdown of RUNX2 protein expression, RUNX2 siRNA treatment of LNCaP-R cells resulted in a significant reduction in cell viability, with survival levels similar to that of the vector-only control cells
(Figures $2 \mathrm{~B}$ and $\mathrm{A}$, respectively). This result suggested that ectopic expression of RUNX2 caused the increased cell viability. Because we showed that bicalutamide treatment increased expression of RUNX2 in vivo (Figures 1A-C), we assessed cell viability following a clinically relevant dose of bicalutamide (Figure 2C). Forty-eight hours after bicalutamide treatment, LNCaP-R cells were significantly more viable than $\mathrm{LNCaP}-\mathrm{V}$ cells; this effect was abrogated by RUNX2 siRNA treatment. As we know that LNCaP tumours treated with bicalutamide exhibit profound hypoxia (Ming et al, 2012), we assessed cell survival following a significant hypoxic insult $\left(72 \mathrm{~h}\right.$ at $\left.0.1 \% \mathrm{O}_{2}\right)$. LNCaP-V cells did not survive the hypoxic conditions; however, although the growth rate of LNCaP-R cells was affected by hypoxia, they recovered and continued to grow (Figure 2D). To support the role of RUNX2 in mediating resistance to chemotherapy, we treated PC3 cells (known to have high endogenous RUNX2 expression; (Akech et al, 2010) with RUNX2 siRNA and found that cells treated with RUNX2 siRNA were significantly more sensitive to docetaxel than ordinary PC3 cells at 25,50 and $100 \mathrm{~nm}$ (Figure $2 \mathrm{E}, P \leqslant 0.05$ ). Moreover, we used hypoxia pre-treatment $\left(0.1 \% \mathrm{O}_{2}\right.$ for $\left.2 \mathrm{~h}\right)$ to induce RUNX2 expression in LNCaP cells, and then measured the sensitivity of these cells to docetaxel with or without siRNA treatment. Pre-treatment with hypoxia resulted in cells being more resistant to docetaxel (Figure 2F); this effect was abolished following treatment with RUNX2 siRNA. In summary, endogenous and forced expression (either through ectopic or environmental modulation) of RUNX2 renders LNCaP cells resistant to docetaxel treatment.
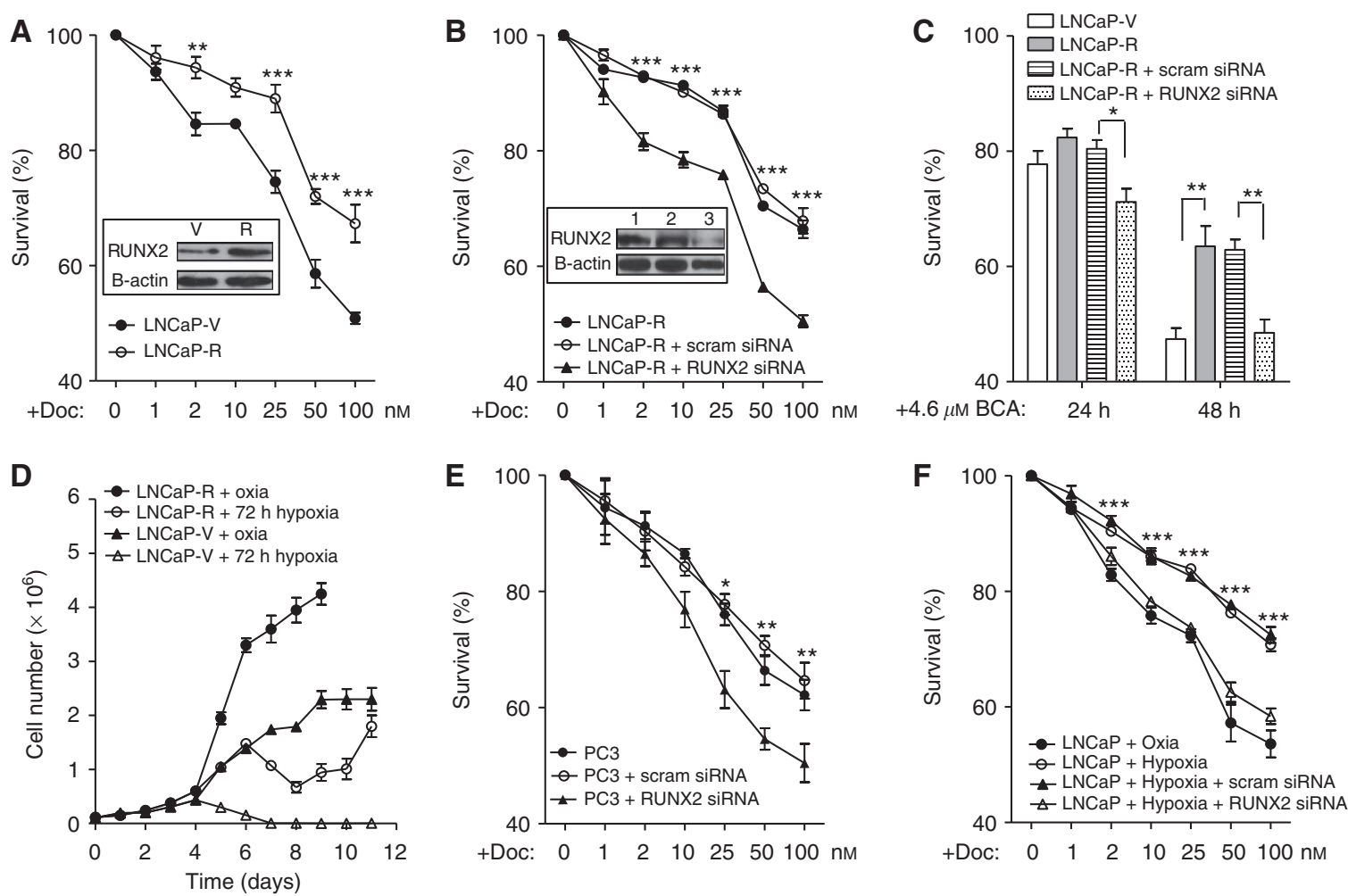

Figure 2 Increased RUNX2 expression results in resistance to docetaxel in vitro. (A) LNCaP-V or LNCaP-R cells were exposed to docetaxel for $48 \mathrm{~h}$ before XTT assay. Results shown are mean \pm s.e. for three independent experiments. Inset is representative western blot showing RUNX2 protein expression in stable cell lines. (B) LNCaP-R cells were treated with either scrambled siRNA or RUNX2 siRNA, and response to docetaxel was measured using an XTT assay after $48 \mathrm{~h}$. Results shown are mean \pm s.e. of three independent experiments. Inset is representative western blot showing RUNX2 expression in I - LNCaP-R cells, 2 - LNCaP-R cells treated with scrambled siRNA and 3 - LNCaP-R cells treated with RUNX2 siRNA. (C) LNCaP cells were treated with scrambled or RUNX2 siRNA and viability following bicalutamide measured after 24 and $48 \mathrm{~h}$ using an XTT assay. Results shown are mean \pm s.e. of three independent experiments. (D) LNCaP-V or LNCaP-R cells were counted using a trypan blue exclusion assay following chronic hypoxia $\left(0.1 \% \mathrm{O}_{2}\right.$ for $72 \mathrm{~h}$ ). Results shown are mean \pm s.e. of three independent experiments. (E) PC3 cells were treated with scrambled siRNA or RUNX2 siRNA, and response to docetaxel was measured using an XTT assay. Results shown are mean \pm s.e. of three independent experiments. (F) LNCaP cells were transfected with scrambled siRNA or RUNX2 siRNA before hypoxia exposure $\left(0.1 \% \mathrm{O}_{2}\right)$ for $2 \mathrm{~h}$ prior to treatment with docetaxel. Cell viability was measured after $48 \mathrm{~h}$ using an $\mathrm{XTT}$ assay. ${ }^{*} P \leqslant 0.05,{ }^{* *} P \leqslant 0.01$ and ${ }^{* * *} P \leqslant 0.001$ (two-way ANOVA). 
RUNX2-mediated resistance to chemotherapy is a result of resistance to apoptosis

We next evaluated if increased survival of RUNX2-overexpressing cells was mediated through alteration of their apoptotic potential. Using a DNA laddering assay, intensity of the DNA banding in the LNCaP-R cells was significantly less than that of the vector-onlyexpressing cells after $48 \mathrm{~h}$ of docetaxel treatment, indicative of reduced apoptosis in the cells (Figure 3A). With the caspase 3/7 assay, significantly more apoptosis was found to occur in the LNCaP-V cells compared with - R cells, with a 2.75 - and 1.85 -fold change, respectively, in the docetaxel-treated over vehicle-onlytreated controls $(P \leqslant 0.05)$ (Figure 3B). Similarly, the TUNEL assay revealed a trend of less apoptosis in the LNCaP-R cells compared with the LNCaP-V cells at all points of treatment investigated, and a significant difference was found at three of the four time points tested (16h $(P \leqslant 0.05) ; 20 \mathrm{~h} \quad(P \leqslant 0.01)$; and $48 \mathrm{~h} \quad(P \leqslant 0.05))$ (Figure $3 \mathrm{C}$ ). Furthermore, $54 \%$ and $33 \%$ less apoptosis was found to occur in the LNCaP-R cells than the LNCaP-V cells after 48 and $72 \mathrm{~h}$ of treatment, respectively, when assayed using a caspase 3 cleavage assay (Figure 3D). Taken together, these results demonstrate that increased expression of RUNX2 in LNCaP cells leads to increased resistance to apoptosis.

\section{RUNX2-associated resistance to apoptosis is mediated through Bcl-2}

To address a requirement for RUNX2 in contributing to a high level of cell survival, we examined the expression of Bcl-2 in our RUNX2-overexpressing LNCaP cells. Levels of Bcl-2 were 2.4-fold

A
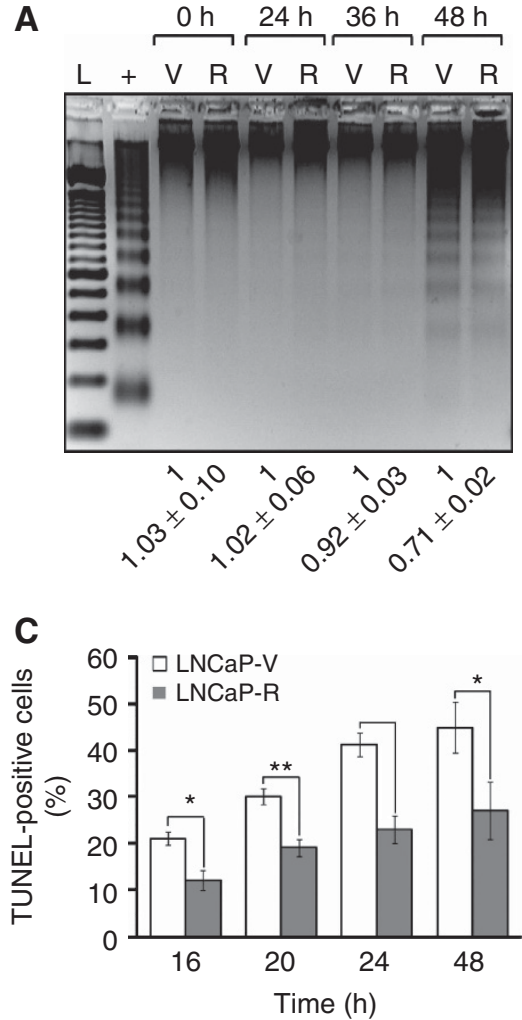

higher in LNCaP-R cells $(P \leqslant 0.01)$, whereas levels of Bax were unaffected (Figure 4A). Examination of the Bcl-2 promoter identified two putative RUNX2-binding sites within $1 \mathrm{~Kb}$ of the transcription start site (Figure 4B). To investigate whether RUNX2 directly regulates $\mathrm{Bcl}-2$ by interacting with and transactivating the $\mathrm{Bcl}-2$ promoter region, luciferase transcriptional activation and ChIP assays were performed. There was a 1.9-fold increase in Bcl-2 promoter activity in LNCaP-R compared with LNCaP-V cells $(P \leqslant 0.05)$; this was abrogated following mutation of the RUNX2binding sites (Figure 4C). As presented in Figure 4D, RUNX2 pull-down was enriched for Bcl-2 promoter fragments compared with IgG-negative controls at both sites. PC3 cells were used as a positive control and showed enhanced binding of RUNX2 with the Bcl-2 promoter. Exposure to hypoxic conditions also increased RUNX2 binding at the Bcl-2 promoter. Moreover, siRNA depletion of RUNX2 in the LNCaP-R cells decreased Bcl-2 mRNA expression by almost $70 \%$ (Figure $4 \mathrm{E}$ ). In addition, we briefly examined the effect of hypoxia on Runx2-mediated Bcl-2 expression, and found that hypoxia $\left(0.1 \% \mathrm{O}_{2}\right.$ for $\left.2 \mathrm{~h}\right)$ increased both Runx2 and Bcl-2 mRNA levels; this effect was partially abrogated by treatment with RUNX2 siRNA (Figure 4F). Overall, these results suggest that the increased resistance to apoptosis evidenced in the RUNX2overexpressing cells is mechanistically linked to RUNX2 upregulation of the anti-apoptotic molecule Bcl-2.

\section{RUNX2 modulates expression of a number of apoptosis-associated genes}

To further delineate the mechanism of RUNX2-mediated resistance to apoptosis, we treated LNCaP-R cells with scrambled or

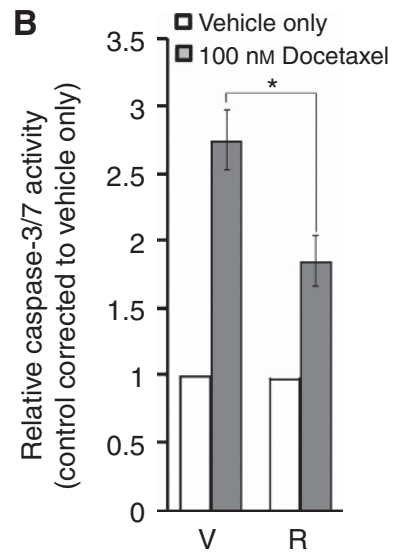

D

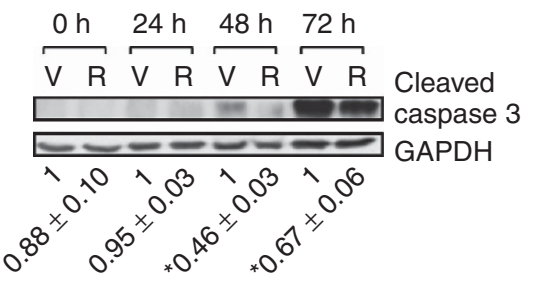

Figure 3 Overexpression of RUNX2 in LNCaP cells causes increased resistance to apoptosis. (A) Following treatment with $100 \mathrm{~nm}$ of docetaxel, DNA from LNCaP-V $(V)$ and LNCaP-R cells $(R)$ was extracted and a DNA laddering assay was carried out. A positive control $(+)$ and I00-bp ladder $(\mathrm{L})$ were also included (representative image shown). Densitometric quantification shown is the result of mean \pm s.e. of three independent experiments. (B) $V$ and $R$ cells were treated for $48 \mathrm{~h}$ before performing caspase 3/7 activity measurements. Results shown are mean \pm s.e. of three independent experiments carried out in triplicate. (C) LNCaP-V and LNCaP-R cells were treated with $100 \mathrm{~nm}$ docetaxel before performing the TUNEL assay as described. Results shown are mean \pm s.e. of five regions of interest for three slides in three independent experiments. (D) Representative immunoblot showing differences in cleaved caspase 3 in $V$ and $R$ cells after docetaxel treatment. Below, densitometric quantification values \pm s.e. for three independent experiments. ${ }^{*} P \leqslant 0.05$ and ${ }^{* *} P \leqslant 0.01$ (Student's $t$-test). 


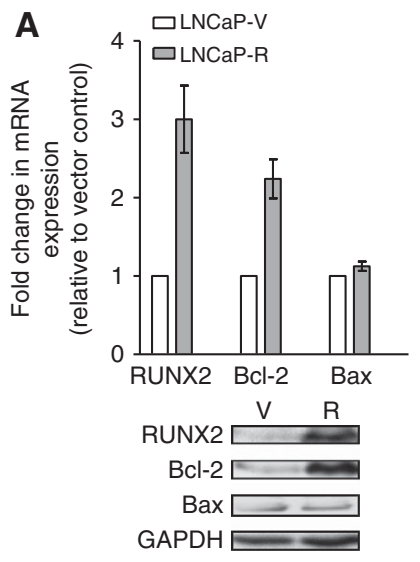

B

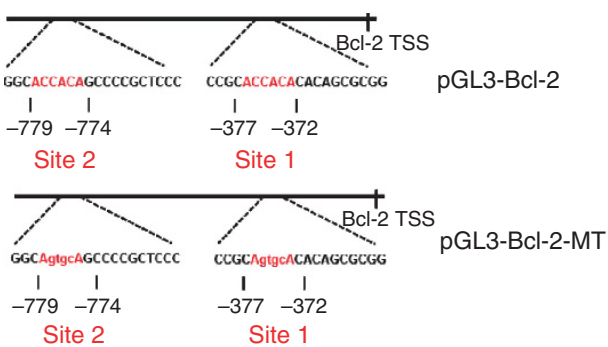

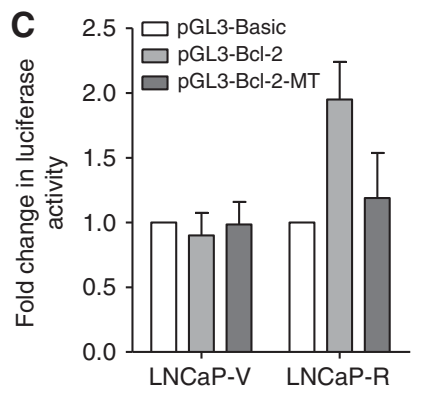
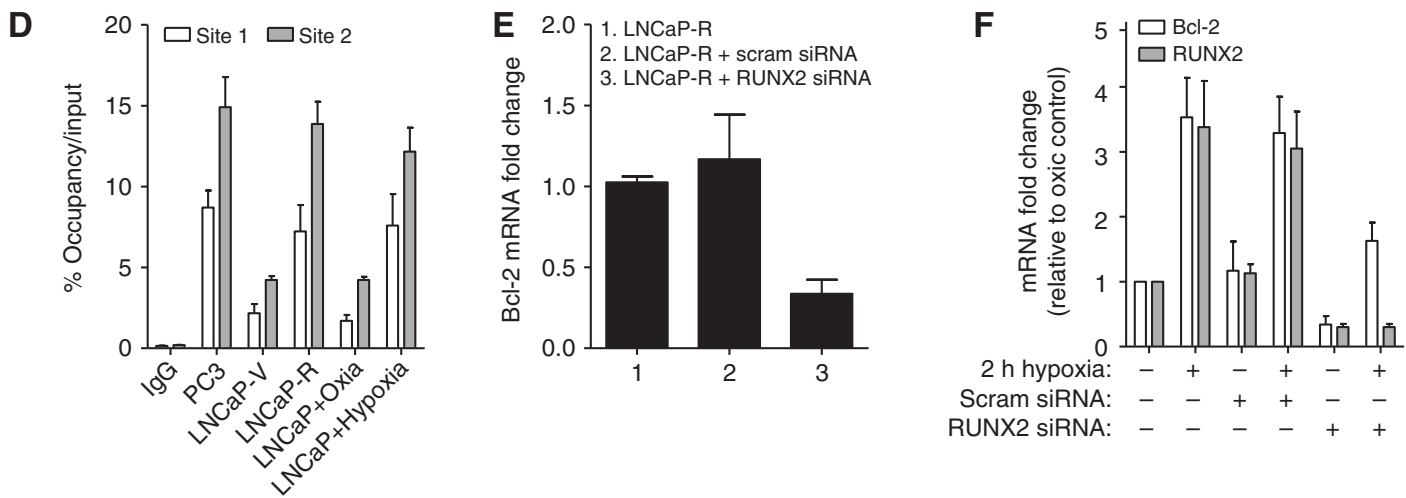

Figure 4 RUNX2 activates the Bcl-2 promoter. (A) RUNX2, Bcl-2 and Bax mRNA levels in LNCaP-V (V) or LNCaP-R (R) cells. Results shown are mean \pm s.e. of three independent experiments. Below, representative western blots show $R U N X 2, B c l-2$ and Bax protein expression in $V$ and $R$ cells. (B) Diagram illustrating two RUNX2-binding sites on the Bcl-2 promoter. Mutated bases in pGL3-Bcl-2-MT vector are indicated in lower case. (C) $V$ or R cells were transfected with either pGL3-basic, pGL3-Bcl-2 or pGL3-Bcl-2-MT vectors before carrying out the luciferase assay All values represent relative firefly luciferase activity normalised to internal and are mean \pm s.e. for three independent experiments. (D) RT-Q-PCR analysis of RUNX2 ChIP assay using primers designed to span RUNX2-binding site I or 2. Results shown are mean \pm s.e. of three independent experiments. (E) LNCaP-R cells (I) were treated with either scrambled siRNA (2) or RUNX2 siRNA (3), and Bcl-2 mRNA levels were assessed. Results shown are mean \pm s.e. of three independent experiments. (F) LNCaP cells were exposed to hypoxia $\left(0.1 \% \mathrm{O}_{2}\right.$ for $\left.2 \mathrm{~h}\right)$ and/or transfected with scrambled or RUNX2 siRNA, and levels of RUNX2 and $\mathrm{Bcl}-2$ mRNA were measured using RT-Q-PCR. Results shown are mean \pm s.e. of three independent experiments.

Bcl-2 siRNA. These cells were then exposed to various doses of docetaxel for $48 \mathrm{~h}$. Although Bcl-2 siRNA resulted in a $75 \%$ reduction of Bcl-2 protein (Figure $5 \mathrm{~A}$ ), survival of cells was not fully decreased to that observed in vector-only cells (Figure 5B). These results suggested that molecules other than Bcl-2 were involved in RUNX2-mediated resistance to apoptosis. Consequently, we extracted RNA from LNCaP-R and -V cells and analysed expression using an apoptosis-specific RT-Q-PCR array. Of the 88 tested genes, 16 pro- and anti-apoptotic genes were significantly differentially expressed in the RUNX2-overexpressing cells, and these effects were reversed in the RUNX2 siRNA-treated LNCaP-R cells (Figure 5C). Analysis, based on published functions of each gene, indicates that increased expression of RUNX2 favours a more pro-survival molecular phenotype.

\section{DISCUSSION}

The anti-androgen bicalutamide is a commonly used treatment for locally advanced prostate cancer (Anderson, 2003). A key finding in this study is the increase of RUNX2 expression during clinically relevant scheduling of bicalutamide. This induction is likely due to bicalutamide-induced hypoxia, as previous studies from our laboratory demonstrated that bicalutamide treatment of LNCaP tumours in vivo resulted in profound and persistent hypoxia
(Ming et al, 2012) and that RUNX2 expression was upregulated in hypoxia-selected LNCaP cells (Butterworth et al, 2008). In vitro analyses revealed that hypoxia increased RUNX2 expression in LNCaP cells, whereas bicalutamide treatment had no effect (Figures 1D and E). These findings have important clinical relevance as it is widely known that hypoxia drives the malignant progression of many cancer types, including prostate cancer (Maxwell et al, 2001).

We have shown that increased RUNX2 expression, whether ectopic or endogenous, provides LNCaP prostate cancer cells with a survival advantage when exposed to docetaxel, bicalutamide or hypoxia. This finding is also clinically relevant because docetaxel is one of the drugs used to treat men who have relapsed after ADT (Mottet et al, 2011). It will be important to understand the consequence of increased RUNX2 expression with respect to a broad spectrum of chemotherapy agents that act through a diverse series of mechanisms with multiple cellular targets. Nevertheless, here we demonstrated that the survival advantage of cells with elevated RUNX2 expression following exposure to docetaxel was because of reduced apoptotic potential mediated at least in part by Bcl-2. We characterised the relationship between RUNX2 and $\mathrm{Bcl}-2$, and established that increased RUNX2 was associated with increased Bcl-2 at both the mRNA and protein level, whereas levels of Bax mRNA and protein were unaffected. We also demonstrated that RUNX2 binds to and transactivates the $\mathrm{Bcl}-2$ promoter, 

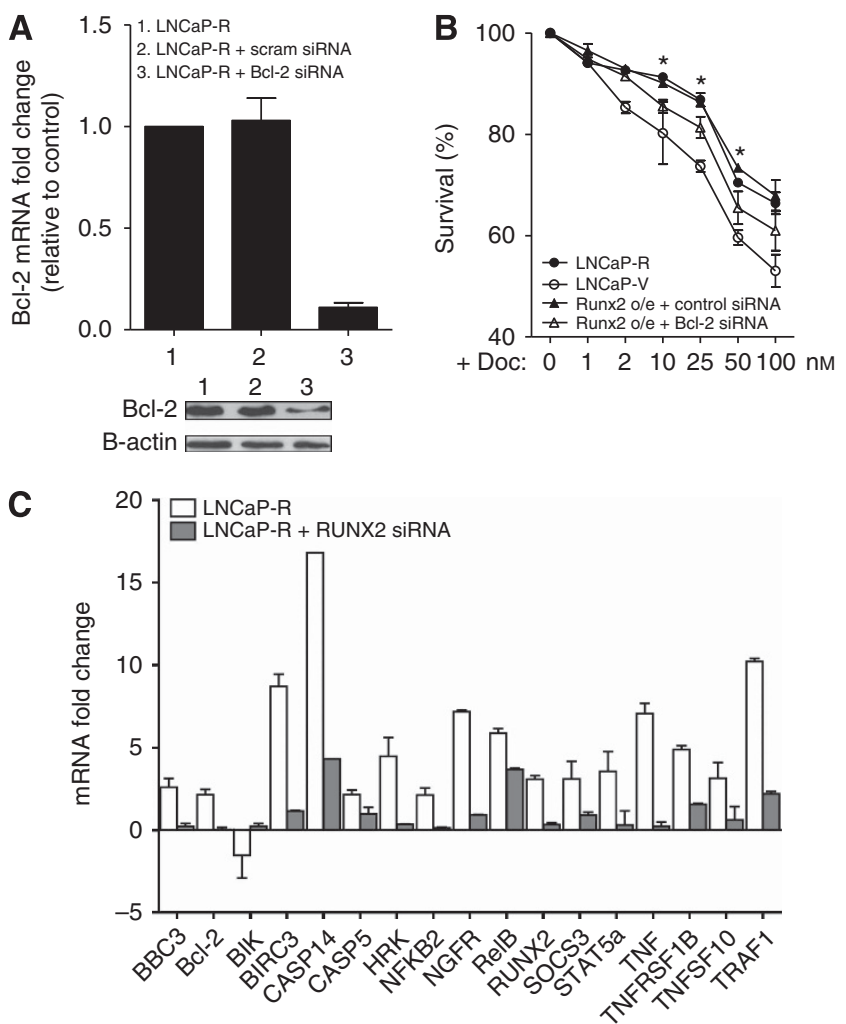

Figure $5 \mathrm{Bcl}-2$ is not the only apoptosis-associated gene activated by RUNX2. (A) Bcl-2 mRNA and protein expression (inset below) of LNCaP$\mathrm{R}$ cells and same cells after treatment with scrambled or Bcl-2 siRNA. Results shown are mean \pm s.e. for three independent experiments. (B) LNCaP-V and LNCaP-R cells treated with scrambled siRNA or Bcl-2 siRNA were exposed docetaxel for $48 \mathrm{~h}$ before cell viability was assessed by XTT assay. Results shown are mean \pm s.e. for three independent experiments. (C) RT-Q-PCR analysis of gene expression in LNCaP-R and LNCaP-R cells pre-treated with RUNX2 siRNA for $24 \mathrm{~h}$. Results shown are mean \pm s.e. of three independent experiments.

providing a mechanism for how RUNX2 affects the apoptotic potential of the cells. Interestingly, in our previous studies with bicalutamide-treated LNCaP tumours (Ming et al, 2012), expression of Bcl-2 mRNA followed a similar pattern to that observed for RUNX2 mRNA in the current study, confirming a relationship between the two. In support of these findings, ectopic expression of RUNX2 in mammary epithelial cells induced expression of Bcl-2 and had a role in the progression of cells to a tumorigenic phenotype (Pratap et al, 2009). Other studies have also shown a correlation between RUNX2 expression and tumour cell survival or tumorigenesis, depending on the cellular context (Zaidi et al, 2007; Chua et al, 2009). Increased Bcl-2 expression is a key hallmark in the malignant progression of prostate cancer, with increased Bcl-2 expression indicative of advanced tumour stage, high Gleason score and a predictor for early disease recurrence after surgery (Revelos et al, 2005). Conversely, recent studies in osteosarcoma cells have shown that although RUNX2 did not affect Bcl-2 expression, RUNX2 did potentiate the expression of Bax, a pro-apoptotic molecule (Eliseev et al, 2008; Goloudina et al, 2012). This divergence suggests that how RUNX2-modulated apoptosis may be cell-type specific, and more research needs to be carried out to confirm the effects of blocking RUNX2 expression in various tumours.

Previous studies have demonstrated a relationship between hypoxia and induction of Bcl-2 (Wang et al, 2002); in the current study, we briefly assessed levels of RUNX2 and Bcl-2 mRNA following hypoxia and RUNX2 siRNA. We found that hypoxiainduced Bcl-2 expression may be partially due to RUNX2 overexpression; although a RUNX2-independent pathway does exist. Further studies are needed to fully understand the intricacies of this complex relationship.

Studies carried out using Bcl-2 siRNA in an XTT assay revealed that Bcl-2 alone cannot account for the difference in RUNX2mediated sensitivity to apoptosis. An apoptosis-specific RT-QPCR array identified a number of pro-and anti-apoptotic genes, which are differentially expressed in RUNX2-overexpressing cells compared with controls. Putative RUNX2-binding sites were present in the promoter region of seven of the genes differentially expressed in our RUNX2-overexpressing cells (Bcl-2, cIAP2, Stat5a, Casp14, TRAIL, Casp5 and PUMA), suggesting that RUNX2 may be involved in directly regulating the expression of other apoptosis-associated genes; however, further studies will be necessary to demonstrate such regulation. Although both pro- and anti-apoptotic factors were found to be increased in RUNX2overexpressing cells, a more apoptosis-resistant phenotype was favoured in this case. It is feasible to suggest that two processes may occur within a population of cancer cells with increased RUNX2 expression; a small number of cells may die due to the proapoptotic signals they receive; however, many more may become apoptosis resistant, resulting in the pro-survival phenotype observed in the RUNX2-overexpressing LNCaP cells. Nonetheless, it is clear that the role of RUNX2 in apoptosis is complex and likely to be context specific, and thus warrants further investigation.

In summary, this study strongly suggests that RUNX2 has a major role in treatment-induced malignant progression of prostate cancer. Data presented indicate that hypoxia, a microenvironmental consequence of ADT, serves to increase RUNX2 expression in tumour cells. Importantly, this study provides mechanistic insight into how RUNX2 interacts with Bcl-2, affecting the ability of cells to undergo normal apoptotic processes in response to a commonly used chemotherapy agent. Greater understanding of the intricacies and modifications of molecules aberrantly regulated in prostate cancer is vital if progress in improving treatment and, ultimately, prevention of the disease is to be achieved. With this in mind, data provided in the current study highlight RUNX2 as a promising therapeutic target with the potential to help control the malignant progression of prostate cancer.

\section{ACKNOWLEDGEMENTS}

Financial support for this work was received from Prostate Cancer Charity (operating code 110857) and NIH grant 5P01CA140043. Bicalutamide was a kind gift from AstraZeneca, UK.

Supplementary Information accompanies the paper on British Journal of Cancer website (http://www.nature.com/bjc)

\section{REFERENCES}

Akech J, Wixted JJ, Bedard K, van der Deen M, Hussain S, Guise TA, van Wijnen AJ, Stein JL, Languino LR, Altieri DC, Pratap J, Keller E, Stein GS, Lian JB (2010) RUNX2 association with progression of prostate cancer in patients: mechanisms mediating bone osteolysis and osteoblastic metastatic lesions. Oncogene 29: 811-821
Alonzi R, Padhani AR, Taylor NJ, Collins DJ, D’Arcy JA, Stirling JJ, Saunders MI, Hoskin PJ (2011) Antivascular effects of neoadjuvant androgen deprivation for prostate cancer: an in vivo human study using susceptibility and relaxivity dynamic MRI. Int J Radiat Oncol Biol Phys 80: $721-727$ 
Anderson J (2003) The role of antiandrogen monotherapy in the treatment of prostate cancer. BJU Int 91: 455-461

Bellido T, Ali AA, Plotkin LI, Fu Q, Gubrij I, Roberson PK, Weinstein RS, O’Brien CA, Manolagas SC, Jilka RL (2003) Proteasomal degradation of RUNX2 shortens parathyroid hormone-induced anti-apoptotic signaling in osteoblasts. A putative explanation for why intermittent administration is needed for bone anabolism. J Biol Chem 278: 50259-50272

Blyth K, Vaillant F, Hanlon L, Mackay N, Bell M, Jenkins A, Neil J, Cameron E (2006) RUNX2 and MYC collaborate in lymphoma development by suppressing apoptotic and growth arrest pathways in vivo. Cancer Res 66: 2195-2201

Bonkhoff H, Berges R (2010) From pathogenesis to prevention of castration resistant prostate cancer. Prostate 70: 100-112

Butterworth KT, McCarthy HO, Devlin A, Ming L, Robson T, McKeown SR, Worthington J (2008) Hypoxia selects for androgen independent LNCaP cells with a more malignant geno- and phenotype. Int J Cancer 123: $760-768$

Cassavaugh J, Lounsbury KM (2011) Hypoxia-mediated biological control. J Cell Biochem 112: 735-744

Chaudary N, Hill RP (2007) Hypoxia and metastasis. Clin Cancer Res 13: 1947-1949

Chua CW, Chiu YT, Yuen HF, Chan KW, Man K, Wang X, Ling MT, Wong YC (2009) Suppression of androgen-independent prostate cancer cell aggressiveness by FTY720: validating RUNX2 as a potential antimetastatic drug screening platform. Clin Cancer Res 15: 4322-4335

Eliseev RA, Dong YF, Sampson E, Zuscik MJ, Schwarz EM, O’Keefe RJ, Rosier RN, Drissi MH (2008) RUNX2-mediated activation of the Bax gene increases osteosarcoma cell sensitivity to apoptosis. Oncogene 27: 3605-3614

Ferlay J, Shin HR, Bray F, Forman D, Mathers C, Parkin DM (2010) Estimates of worldwide burden of cancer in 2008: GLOBOCAN 2008. Int J Cancer 127: 2893-2917

Goloudina AR, Tanoue K, Hammann A, Fourmaux E, Le Guezennec X, Bulavin DV, Mazur SJ, Appella E, Garrido C, Demidor ON (2012) Wip1 promotes RUNX2-dependent apoptosis in p53-negative tumours and protects normal tissues during treatment with anticancer agents. Proc Natl Acad Sci USA 109: 68-75

Hanahan D, Weinberg RA (2011) Hallmarks of cancer: the next generation. Cell 144: 646-674

Kwon TG, Zhao X, Yang Q, Li Y, Ge C, Zhao G, Franeschi RT (2011) Physical and functional interactions between RUNX2 and HIF-1alpha induce vascular endothelial growth factor gene expression. J Cell Biochem 112: 3582-3593

Leong DT, Lim J, Goh X, Pratap J, Pereira BP, Kwok HS, Nathan SS, Dobson JR, Lian JB, Ito Y, Voorhoeve PM, Stein GS, Salto-Tellez M, Cool SM, van Wignen AJ (2010) Cancer-related ectopic expression of the bone-related transcription factor RUNX2 in non-osseous metastatic tumour cells is linked to cell proliferation and motility. Breast Cancer Res 12: R89
Majmundar AJ, Wong WJ, Simon MC (2010) Hypoxia-inducible factors and the response to hypoxic stress. Mol Cell 40: 294-309

Maxwell PH, Pugh CW, Ratcliffe PJ (2001) Activation of the HIF pathway in cancer. Curr Opin Genet Dev 11: 293-299

Ming L, Byrne N, Camac S-N, Mitchell C, Ward C, Waugh D, McKeown SR, Worthington J (2012) Androgen deprivation results in time-dependent hypoxia in LNCaP prostate tumours; informed scheduling of the bioreductive drug $\mathrm{AQ} 4 \mathrm{~N}$ improves treatment response. Int J Cancer; e-pub ahead of print; doi:10.1002/ijc.27796

Mottet N, Bellmunt J, Bolla M, Joniau S, Mason M, Matveev V, Schmid H-P, Van der Kwast T, Wiegel T, Zattoni F, Heidenreich A (2011) EAU guidelines on prostate cancer. Part II: Treatment of advanced, relapsing, and castration-resistant prostate cancer. Eur Urol 59: 572-583

Pratap J, Imbalzano K, Underwood J, Cohet N, Gokul KD, Akech J, van Wijnen AJ, Stein JL, Imbalzano AN, Nickerson JA, Lian JB, Stein GS (2009) Ectopic RUNX2 expression in mammary epithelial cells disrupts formation of normal acini structure: Implications for breast cancer progression. Cancer Res 69: 6807-6814

Pratap J, Lian JB, Javed A, Barnes GL, van Wijnen AJ, Stein JL, Stein GS (2006) Regulatory roles of RUNX2 in metastatic tumour and cancer cell interactions with bone. Cancer Metastasis Rev 25: 589-600

Revelos K, Petraki C, Gregorakis A, Scorilas A, Papanastasiou P, Koutsilieris $M$ (2005) Immunohistochemical expression of $\mathrm{Bcl} 2$ is an independent predictor of time-to-biochemical failure in patients with clinically localized prostate cancer following radical prostatectomy. Anticancer Res 25: 3123-3133

Tamiya H, Ikeda T, Jeong JH, Saito T, Yano F, Jung YK, Ohba S, Kawaquchi $\mathrm{H}$, Chung UI, Choi JY (2008) Analysis of the RUNX2 promoter in osseous and non-osseous cells and identification of HIF2A as a potent transcription activator. Gene 416: 53-60

Wang JH, Wu QD, Bouchier-Hayes D, Redmond HP (2002) Hypoxia upregulates Bcl-2 expression and suppresses interferon-gamma induced antiangiogenic activity in human tumor derived endothelial cells. Cancer 94: 2745-2755

Workman P, Aboagye EO, Balkwill F, Balmain A, Bruder G, Chaplin DJ, Double JA, Everitt J, Farningham DA, Glennie MJ, Kelland LR, Robinson V, Stratford IJ, Tozer GM, Watson S, Wedge SR, Eccle SA (2010) Guidelines for the welfare and use of animals in cancer research. $\mathrm{Br} J$ Cancer 102: 1555-1577

Zaidi SK, Pande S, Pratap J, Gaur T, Grigoriu S, Ali SA, Stein JL, Lian JB, van Wijnen AJ, Stein GS (2007) RUNX2 deficiency and defective subnuclear targeting bypass senescence to promote immortalization and tumourigenic potential. Proc Natl Acad Sci USA 104: 19861-19866

Zhang H, Pan Y, Zheng L, Choe C, Lindgren B, Jensen ED, Westerndorf JJ, Cheng L, Huang $\mathrm{H}$ (2011) FOXO1 inhibits RUNX2 transcriptional activity and prostate cancer cell migration and invasion. Cancer Res 71: $3257-3267$

This work is published under the standard license to publish agreement. After 12 months the work will become freely available and the license terms will switch to a Creative Commons Attribution-NonCommercial-Share Alike 3.0 Unported License. 